\title{
Caught in the act: A helium-reionizing quasar near the line of sight to Q0302-003 ${ }^{\star}$
}

\author{
P. Jakobsen ${ }^{1}$, R. A. Jansen ${ }^{1,2}$, S. Wagner ${ }^{3}$, and D. Reimers ${ }^{4}$ \\ 1 Astrophysics Division, RSSD, European Space Agency, ESTEC, 2200 AG Noordwijk, The Netherlands \\ 2 Department of Physics and Astronomy, Arizona State University, Tempe AZ 85287-1504, USA \\ 3 Landessternwarte Heidelberg, Königstuhl 12, 69117 Heidelberg, Germany \\ ${ }^{4}$ Hamburger Sternwarte, Gojenbergsweg 112, 21029 Hamburg, Germany
}

Received 23 October 2002 / Accepted 30 October 2002

\begin{abstract}
We report the discovery of a quasar at $z=3.050 \pm 0.003$, closely coincident in redshift with the isolated lowopacity feature seen near $z \simeq 3.056$ in the otherwise black portion of the He II Gunn-Peterson absorption trough seen toward the $z=3.286$ background quasar Q0302-003, located 6.5 away on the sky. We explore plausible models for the He III ionization zone created by this neighboring quasar and its interception with the line of sight toward Q0302-003. At its present brightness of $V \simeq 20.5$ and separation of $D_{\perp} \simeq 3.2 \mathrm{Mpc}$, the quasar can readily account for the opacity gap in the He II absorption spectrum of Q0302-003, provided it has been active for $t_{Q} \gtrsim 10^{7} \mathrm{y}$. This is the first clear example of the "transverse" proximity effect and the association of a quasar with its imprint on the intervening absorption detected along an adjacent line of sight.
\end{abstract}

Key words. quasars: individual: QSO 03020-0014, Q0302-003 - quasars: absorption lines - intergalactic medium

\section{Introduction}

One of the more unique contributions of UV space astronomy to observational cosmology has been the first detections of redshifted intervening singly ionized He II Gunn-Peterson absorption due to the Lyman forest and diffuse intergalactic medium (IGM) in the far-UV spectra of high redshift quasars obtained with HST (Jakobsen et al. 1994; Tytler et al. 1995; Reimers et al. 1997), HUT (Davidsen et al. 1996) and FUSE (Kriss et al. 2001). These detections of singly ionized intergalactic helium have not only confirmed qualitatively a fundamental tenet of Big Bang nucleosynthesis theory, but also provided direct support for the long-standing picture of a photoionized intergalactic medium that was reionized by quasars and young galaxies.

Although He II absorption has to date still only been observed toward a total of four high redshift quasars, the dramatic change in character and strength of the He II opacity with redshift strongly suggests that the final stage of reionization of the universe from He II to He III may have occurred at $z \simeq 2.9$; i.e. considerably later than hydrogen reionization which recent evidence suggests may have taken place at $z \simeq 6-7$ (Becker et al. 2001; Djorgovski et al. 2001; Pentericci et al. 2002). Taken together, these results imply that the hydrogen content of the IGM was reionized early by sources having a relatively soft ionizing spectrum (i.e. the first generations of stars), whereas

Send offprint requests to: P. Jakobsen,

e-mail: pjakobsen@rssd.esa.int

* Based on observations collected at the European Southern Observatory, Chile (Proposals 66.A-0307 and 68.A-0194). helium reionization was only completed later once the more energetic quasars and AGNs had formed at $z \lesssim 3$.

The He II absorption at redshifts $z \gtrsim 2.8$ in the three lines of sight probed so far at the resolution and sensitivity of HST/STIS (Q0302-003 ( $z=3.29)$, PKS 1935-692 ( $z=3.18)$ and HE 2347-4342 $(z=2.89))$ all display an underlying trough of seemingly black $\left(\tau_{\mathrm{He} \text { II }}>3\right)$ absorption interrupted by one or more isolated megaparsec-sized "voids" or "gaps" of finite $\left(\tau_{\mathrm{He} \text { II }} \sim 1\right)$ opacity material (Reimers et al. 1997; Anderson et al. 1999; Heap et al. 2000; Smette et al. 2002). This is in marked contrast to the He II absorption at redshifts $z \lesssim 2.8$ observed with HUT toward HS $1700+642(z=2.72)$ and FUSE toward HE 2347-4342, which is much weaker and matches that of the H I Lyman forest more closely (Davidsen et al. 1996; Kriss et al. 2001).

As discussed in detail by Reimers et al. (1997), Heap et al. (2000) and Smette et al. (2002), the isolated He II opacity gaps seen at $z \gtrsim 2.8$ correspond closely to clearings seen in the $\mathrm{H}_{\mathrm{I}}$ Lyman forest, and are naturally interpreted in the conventional quasar reionization picture as the not yet overlapping $\mathrm{He}$ III ionized regions surrounding other foreground quasars or AGNs located near the line of sight.

The four He II voids seen toward Q0302-003, HE 2347-4342, and PKS 1935-692 all appear at redshifts between $z \simeq 2.81$ and $z \simeq 3.10$, and display widths in the range $\Delta z \simeq 0.01-0.02$. In the currently fashionable $H_{0}=65 \mathrm{~km} \mathrm{~s}^{-1} \mathrm{Mpc}^{-1}, \Omega_{0}=0.3, \Lambda_{0}=0.7$ cosmology, these voids extend distances of $D_{\|} \simeq 2-5 \mathrm{Mpc}$ along the line of sight, corresponding to transverse angular distances on the sky 


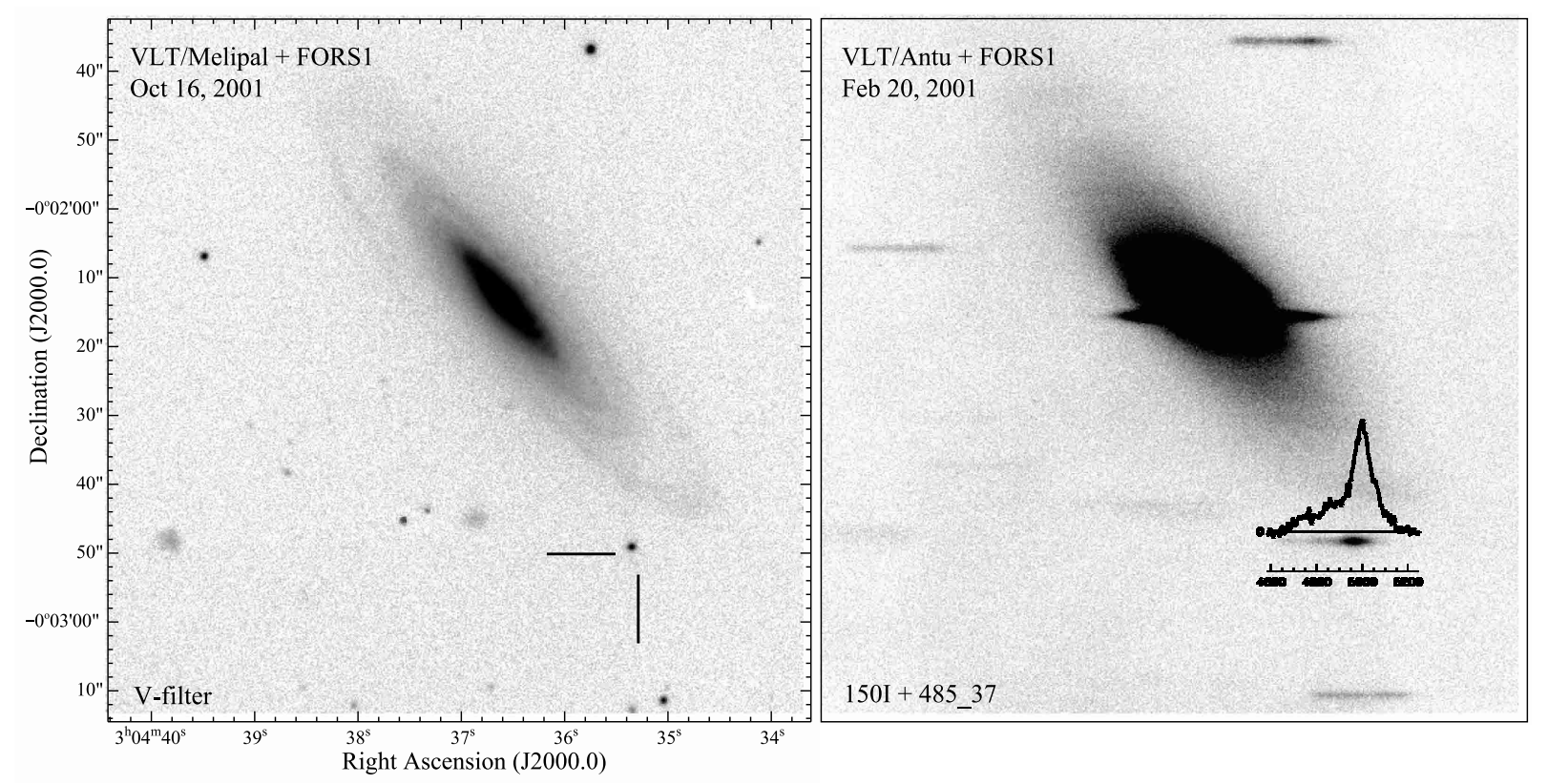

Fig. 1. 1:7 $\times 1: 7$ portion of a $120 \mathrm{~s}$ VLT/FORS $V$-filter image (left) and the corresponding segment of the $1665 \mathrm{~s}$ filtered slitless spectrographic exposure (right) showing the location and discovery spectrum of QSO 03020-0014, located 6.5 northwest of Q0302-003. The 150I grism crossed with the $485 \_37$ filter was used to isolate Ly $\alpha$ in the redshift range $2.8<z<3.2$. The bright $B \simeq 15.5$ Sb galaxy in the foreground is MCG+00-08-082.

in the range $\theta \simeq 5^{\prime}-10^{\prime}$. That this angle is comparable to the field of view of available multi-object spectrographs, makes it obvious to attempt to search for these putative helium-ionizing foreground quasars by spectroscopic means.

In this paper we describe such a search of the field surrounding Q0302-003 using the Focal Reducer/Low Dispersion Spectrograph (FORS) on the ESO VLT, and the resulting discovery of a nearby quasar closely coincident in redshift with the isolated He II low opacity feature seen at $z \simeq 3.056$ toward that object.

\section{Observations}

Our approach was to search for quasar candidates at the redshift of interest through their Ly $\alpha$ emission by imaging the surrounding field of Q0302-003 using VLT/FORS (Seifert et al. 2000) in a slitless spectroscopic mode. The He II low-opacity gap in Q0302-003 is confined to the redshift range $z \simeq 3.05-3.07$ (Heap et al. 2000; Fig. 3). We therefore employed the 150I (5.52 ̊/pixel) low resolution grism crossed with the 486_37 (4600-5100 $\AA$ ) intermediate band filter to isolate Ly $\alpha$ over the range $2.8<z<3.2$ (see Böhnhardt 2001 for details of the FORS observing modes). The use of a filter in addition to the dispersing element had the dual advantage of reducing the risk of crowding by limiting the length of the individual spectra while cutting down the (effectively undispersed) sky background in the slitless exposures.

Our objective was to explore a $13^{\prime} \times 13^{\prime}$ wide field centered on Q0302-003 by means of a $2 \times 2$ pattern of overlapping slitless exposures of $1800 \mathrm{~s}$ duration. The first observations were carried out in Service Mode with FORS1 on UT1/Antu between January 25 and February 20, 2001
(Program 66.A-0307A). The bulk of these data were taken under less-than-ideal conditions of bright sky, high airmass (1.4-2.2) and mediocre seeing (1.1-2.4"), and therefore only reached down to magnitude $V \simeq 22$. Nevertheless, one of the four pointings readily revealed the presence of a $V \simeq$ 20.5 object displaying a broad emission line at the position $\alpha=03^{\mathrm{h}} 04^{\mathrm{m}} 35^{\mathrm{s}} .37 \delta=-00^{\circ} 02^{\prime} 50{ }^{\prime} 9$ (J2000), 6.5 northwest of Q0302-003 (Fig. 1).

Follow-up spectroscopy of this candidate was obtained with FORS1 on UT3/Melipal in Visitor Mode on October 15, 2001 (Programme 68.A-0194A). Figure 2 shows the confirmation spectrum obtained in $3000 \mathrm{~s}$ with the $300 \mathrm{~V}(2.69 \AA /$ pixel $)$ grism and a $1^{\prime \prime}$ wide slit. The spectrum was wavelength calibrated against the standard $\mathrm{He} / \mathrm{Ne} / \mathrm{Ar} / \mathrm{HgCd}$ arc lamps of FORS 1 to an accuracy of $\sigma_{\lambda} \simeq 0.5 \AA$, and photometrically calibrated against the standard star LTT7987 (Hamuy et al. 1994). The spectrum confirms that the object is a high redshift quasar, and that the broad emission line detected in the slitless discovery exposure is indeed $\operatorname{Ly} \alpha$ (Table 1). In accord with current IAU conventions (and to better distinguish it from Q0302-003 typographically), we denote this object QSO 03020-0014 in the following ${ }^{1}$.

Several intervening absorption line systems can be identified in our spectrum of QSO 03020-0014. A resolved Mg II doublet matching several Fe II lines at shorter wavelength is clearly seen at $z_{\mathrm{abs}}=1.298$. A second weaker C Iv system is probably present at $z_{\mathrm{abs}}=2.910$. Of more interest to the problem at hand, however, is the strong $z_{\mathrm{abs}} \approx z_{\mathrm{em}}$

\footnotetext{
${ }^{1}$ While preparing this paper, we noted that QSO 03020-0014 is listed as a point source in the Early Data Release of the Sloan Digital Sky Survey (Object ID 1970729400402035).
} 


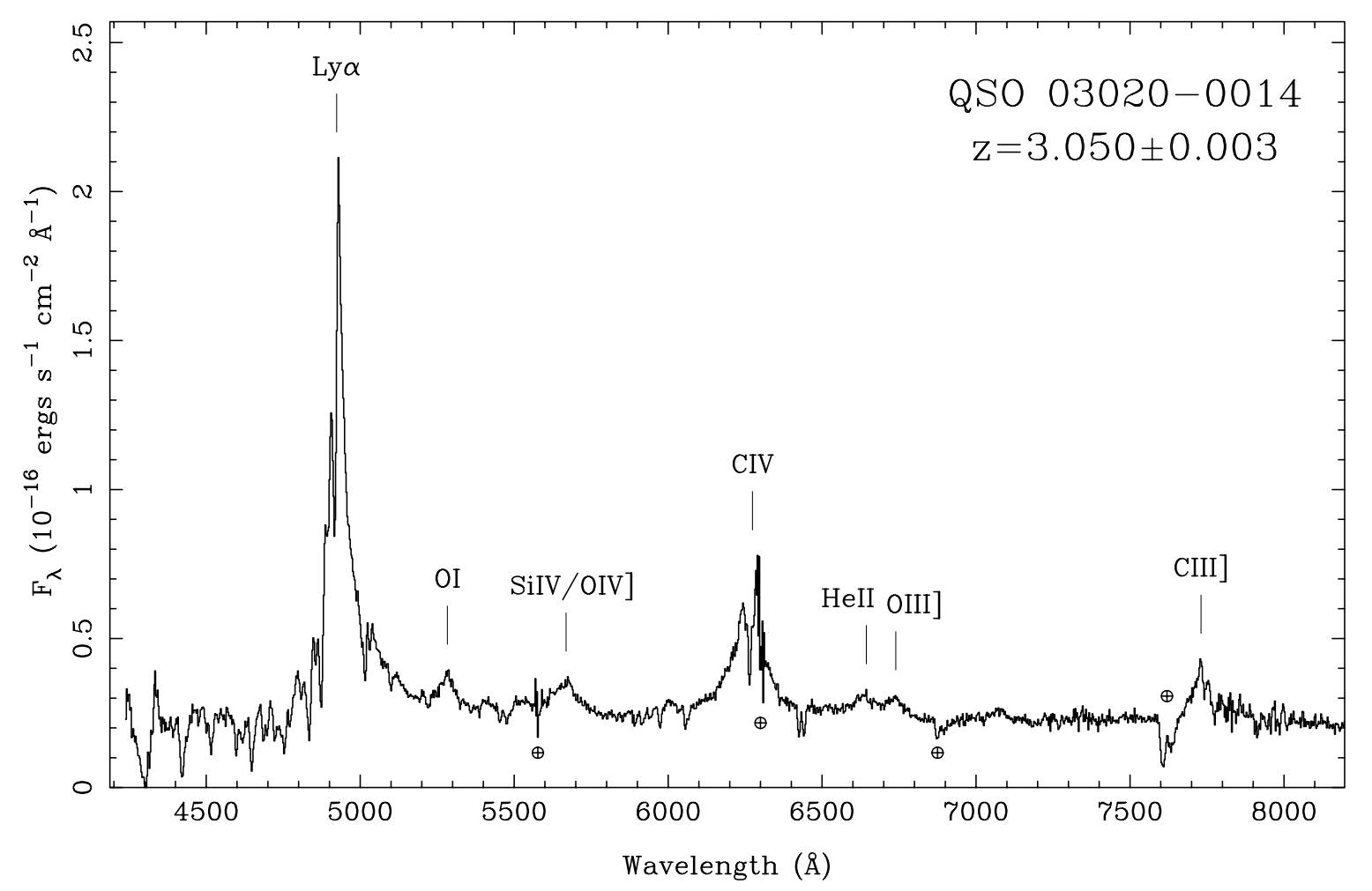

Fig. 2. VLT/FORS spectrum of QSO 03020-0014 (300 V grism, $3000 \mathrm{~s}$ exposure). Emission lines and major atmospheric artifacts in the spectrum are indicated.

Table 1. Emission lines in QSO 03020-0014.

\begin{tabular}{lcc}
\hline \hline \multicolumn{1}{c}{ ID } & Wavelength $(\AA)$ & $z$ \\
\hline Ly $\alpha$ & $4925 \pm 15$ & $3.051 \pm 0.012$ \\
$\mathrm{O}_{\text {I }}$ & $5283 \pm 3$ & $3.050 \pm 0.002$ \\
$\left.\mathrm{Si}_{\text {IV }} / \mathrm{O}_{\text {IV }}\right]$ & $5671 \pm 15$ & $3.052 \pm 0.011$ \\
$\mathrm{C}_{\text {IV }}$ & $6265 \pm 15$ & $3.044 \pm 0.010$ \\
$\mathrm{He}_{\text {II }}$ & $6643 \pm 7$ & $3.050 \pm 0.004$ \\
$\mathrm{O}_{\text {III }}$ & $6738 \pm 7$ & $3.049 \pm 0.004$ \\
$\mathrm{C}_{\text {III }}$ & $7728 \pm 10$ & $3.049 \pm 0.005$ \\
\hline
\end{tabular}

absorption in $\operatorname{Ly} \alpha, \mathrm{N} v$ and $\mathrm{C}$ Iv evident in Fig. 2. This classical high ionization system appears at $z_{\text {abs }} \approx 3.046-3.048, \Delta v \simeq$ $-150-300 \mathrm{~km} \mathrm{~s}^{-1}$ shortward of the emission line redshift.

The presence of the $z_{\mathrm{abs}} \approx z_{\mathrm{em}}$ system (and the telluric absorption affecting the $\left.\mathrm{C}_{\mathrm{III}}\right]$ line) hampers the determination of the precise redshift of QSO 03020-0014. This is reflected in the large uncertainties listed for several of the emission lines in Table 1. Our adopted redshift of $z=3.050 \pm 0.003$ is heavily weighted toward the value indicated by the welldetected O I $\lambda 1304$ line. This choice is also well motivated astrophysically since this low-ionization line does not suffer from the well-known systematic blueshifts exhibited by the higher excitation Ly $\alpha, \mathrm{C}$ IV, Si IV/O IV] and C III] lines and therefore provides the best estimate of the true systemic redshift of QSO 03020-0014 (Gaskell 1982; Tytler \& Fan 1992; McIntosh et al. 1999).

With the aim of obtaining an accurate measure of the brightness of QSO 03020-0014, direct imaging of the object was carried out in the FORS1 Bessel $B, V$ and $R$ filters.
These data were calibrated against the Landolt (1992) standard fields Mark-A and SA98-670, yielding $B=21.51 \pm 0.05$, $V=20.51 \pm 0.05$ and $R=20.20 \pm 0.04$, in agreement with our spectrophotometric calibration.

The initial January/February 2001 Service Mode data also uncovered an at the time undocumented vignetting issue with FORS in slitless mode that results in a usable field of view of $5.3 \times 6.8$ for our particular setup, compared to the nominal 6'.8 $\times 6$ '.8 assumed. Consequently, a vertical $\simeq 2^{\prime}$ wide strip of sky was missed in the $2 \times 2$ pattern of pointings used to map the field surrounding Q0302-003. For completeness, this gap was patched through two additional slitless FORS exposures during the October 2001 Visitor Mode observing run. These observations were obtained under very good observing conditions (seeing $0.5-0.6^{\prime \prime}$ ), and consequently reach approximately a magnitude deeper than the initial Service Mode observations. The supplementary slitless exposures uncovered a second faint broad emission line object in the field at the position $\alpha=03^{\mathrm{h}} 04^{\mathrm{m}} 45^{\mathrm{s}} .94 \delta=-00^{\circ} 11^{\prime} 38^{\prime \prime} .2$ (J2000). However, real time follow-up spectroscopy revealed the object to be a foreground quasar (QSO 03022-0023) at the "uninteresting" redshift $z \simeq 2.14$ ( $\mathrm{C}$ IV in band).

\section{Discussion}

\subsection{Coincidence in redshift}

In Fig. 3, the location of QSO 03020-0014 in redshift space is compared to the detailed profile of the He II opacity gap seen at $z \simeq 3.056$ toward Q0302-003. The shown segment of the normalized absorption spectrum is based on our own reduction 


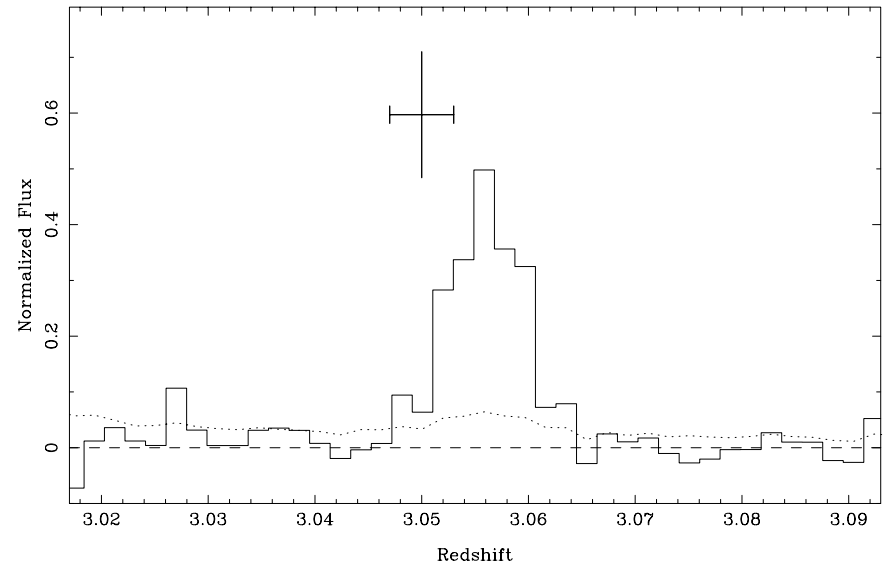

Fig. 3. Comparison in redshift between the measured emission redshift of QSO 03020-0014 (cross) and the isolated low opacity He II feature at $z \simeq 3.056$ seen in the STIS spectrum of Q0302-003 (binned full line). The dotted curve denotes the $1 \sigma$ statistical uncertainty per pixel of the STIS spectrum.

of the STIS observations of Heap et al. (2000). Our profile of the He II feature is closely similar to that published by Heap et al. (2000), but does have slightly sharper edges. Following Heap et al. (2000) we assumed the continuum to be at $F_{\lambda} \simeq$ $2.1 \times 10^{-16} \mathrm{erg} \mathrm{s}^{-1} \mathrm{~cm}^{-2} \AA^{-1}$ when normalizing the spectrum.

It is evident that our measured redshift of $z=3.050 \pm$ 0.003 places QSO 03020-0014 about $\Delta z \simeq 0.006$ shortward of the peak of the opacity gap near its blue edge. Given the low surface density of quasars at $z \sim 3$, this close coincidence in redshift is remarkable, and strongly suggests a causal connection between the two.

Using the quasar luminosity function of Pei (1995), we calculate that the chance probability of a quasar brighter than $V \simeq 20.5$ having a redshift within $\Delta z \lesssim 0.006$ of the peak of the He II feature being located within 6.5 of Q0302-003 is $p \simeq 1 \times 10^{-3}$.

More conservatively, our two slitless spectroscopic observation runs together covered a $13^{\prime} \times 13^{\prime}$ area surrounding Q0302-003; just under half of which was surveyed to a depth of $V \simeq 23$ and the remainder to $V \simeq 22$. The a priori probability of our finding a quasar at $3.04<z<3.08$ anywhere within the area surveyed to this depth is $p \simeq 3 \times 10^{-2}$.

As a consistency check, we note that the total number of quasars found through our slitless observations is in good accord with expectations. The 4600-5100 ̊ filter employed in our observations is capable of detecting high redshift quasars in Ly $\alpha$ over the range $2.8<z<3.2$; in $\mathrm{C}$ IV over the range $2.0<z<2.3$; and in $\mathrm{C}$ III] over the range $1.4<z<1.7$. The predicted number of quasars of these three categories in our exposures are, respectively, $\simeq 0.7, \simeq 0.5$ and $\simeq 0.2$, which is entirely consistent with the one $\operatorname{Ly} \alpha$ and one $\mathrm{C}$ IV quasar actually detected.

In other words, the successful result of our search of the field surrounding Q0302-003 needs to be gauged against the $p \simeq 50 \%$ a priori probability of our having detected a Ly $\alpha$ quasar anywhere in our band, compounded by the $p \simeq 9 \%$ probability that the object falls within $\Delta z \simeq \pm 0.02$ of the peak of the opacity gap of interest.

The above considerations suggest that the null hypothesis that the close coincidence in redshift between QSO 03020-0014 and the He II absorption gap seen in the spectrum of Q0302-003 is merely a chance occurrence can be rejected at the $\gtrsim 95 \%$ confidence level.

\subsection{Astrophysical interpretation}

In their discovery paper, Heap et al. (2000) discuss the reasons for believing that the He II opacity gap seen in the STIS spectrum of Q0302-003 is not merely a statistical fluctuation in the IGM, but caused by a nearby source of He II-ionizing photons. In this section, we explore the astrophysical implications of presuming that QSO 03020-0014 is this source ${ }^{2}$. Throughout the discussion, we assume a cosmological model with $H_{0}=65 \mathrm{~km} \mathrm{~s}^{-1} \mathrm{Mpc}^{-1}, \Omega_{0}=0.3, \Lambda_{0}=0.7$, together with a baryonic density of $\Omega_{\mathrm{b}} h^{2}=0.019$ and a helium abundance by number of $[\mathrm{He} / \mathrm{H}]=0.08$. In this cosmology a redshift difference $\Delta z$ corresponds to a physical distance along the line of sight at redshift $z$ given by

$D_{\|}(z)=\frac{c}{H_{0}} \frac{\Delta z}{(1+z)\left(\Omega_{0}(1+z)^{3}+\Lambda_{0}\right)^{\frac{1}{2}}}$

and an angular separation $\theta$ corresponds to a projected distance

$D_{\perp}(z)=\theta D_{\mathrm{L}}(z)(1+z)^{-2}$

where $D_{\mathrm{L}}$ is the luminosity distance. A convenient approximate analytical expression for $D_{\mathrm{L}}$ in flat lambda cosmologies is given by Pen (1999). All distances and physical parameters quoted below refer to the epoch $z=3.05$.

The angular separation between QSO 03020-0014 and Q0302-003 of $\theta=6.5$ corresponds to a distance $D_{\perp} \simeq$ $3.2 \mathrm{Mpc}$ between QSO 03020-0014 ( $\left.D_{\mathrm{L}} \simeq 27.9 \mathrm{Gpc}\right)$ and the line of sight to Q0302-003. If ionizing radiation emitted by QSO 03020-0014 is responsible for creating the He II void, it immediately follows that the quasar must have been on for at least

$t_{\mathrm{Q}}>D_{\perp} / c \simeq 1 \times 10^{7} \mathrm{yr}$

prior to our observing it at $z \simeq 3.05$. This is consistent with current estimates of the typical quasar lifetime, which from other considerations is inferred to be of the order $10^{7} \lesssim t_{\mathrm{Q}} \lesssim$ $10^{8}$ yr (Martini \& Weinberg 2001; Haiman \& Hui 2001; Barger et al. 2001 and references therein). The above dimension for the He III region surrounding QSO 03020-0014, and the corresponding constraint on the quasar lifetime, are similar to those inferred from the analysis of the "proximity effect" detected in the He II absorption toward Q0302-003 and PKS1935-692 caused by the ionizing radiation emitted by the background quasars themselves (Hogan et al. 1997; Anderson et al. 1999).

\footnotetext{
2 Although galaxies are likely to be feeble sources of $E>54 \mathrm{eV}$ photons compared to quasars, we note that the deep survey of Lyman Break galaxies in the Q0302-003 field carried out by Adelberger et al. (2002) did not reveal any such objects near the $z \simeq 3.06$ redshift of the gap.
} 


\subsection{Photoionization models}

Heap et al. (2000) proposed a simple photoionization model for the He II absorption spectrum of Q0302-003 and its opacity gap. This model was further developed by Smette et al. (2002), who applied it to the two similar absorption gaps seen in the spectrum of HE 2347-4342. The model starts with the conventional picture of an intergalactic medium in photoionization equilibrium with an ionizing background that extends to He II-ionizing photon energies $E>54 \mathrm{eV}$. The effect of an ionizing source located near the line of sight is treated as a localized enhancement of the ionizing background whose intensity decreases as $R^{-2}$ with distance from the object. By adjusting the assumed spectrum of the ionizing background, the relative brightness and hardness of the spectrum of the adjacent quasar, and its distance from the line of sight, the observed width and contrast of a given He II gap can be reproduced.

Smette et al. (2002) also assumed that the initial He II optical depth in the vicinity of an opacity gap is $\tau_{\text {He II }}^{0} \simeq 4.5$, which is akin to assuming that the helium content of the IGM at $z \sim 3$ is already pre-ionized to the level $\mathrm{He}$ II/He III $\sim \mathrm{He}$ II $/ \mathrm{He} \sim$ $10^{-2}$. Our examination of the available STIS data has convinced us that the He II observations do not require that this necessarily be the case, and that the observations are equally consistent with the significantly larger values of $\tau_{\mathrm{He} \mathrm{II}} \approx 10-100$ expected in the delayed He II reionization scenario. In particular, the high opacity He II troughs surrounding the opacity gaps at $z \gtrsim 2.8$ toward Q0302-003, HE 2347-4342, and PKS 1935-692 are in our view all consistent with being truly black; i.e. show no sign of a statistically significant residual flux having been detected in their dark portions ${ }^{3}$. This, of course, does not prove that the true He II optical depth at $z \gtrsim 2.8$ is not, in fact, lurking at $\tau_{\text {He II }} \simeq 4.5$ just below the STIS detection threshold as assumed by Smette et al. (2002). However, if the reduction in absorbing power due to forest structure of the IGM is taken into account, such a large He II optical depth may be difficult to reconcile with a conventional intergalactic helium abundance and low baryon density, unless the bulk of the intergalactic helium is in the form of He II at $z \gtrsim 2.8$ (Jakobsen 1998). Furthermore, the proposition that He II was reionized by $z \sim 3$ implicitly requires the existence of a population of faint energetic $E>54 \mathrm{eV}$ sources having a sufficiently large space density to overcome the extremely short mean path for $E>54 \mathrm{eV}$ photons implied by the observed strength of the He II opacity at $z \sim 3$ (MiraldaEscudé et al. 2000).

As we shall argue below, our detection of QSO 03020-0014 does not directly address this issue, except in the sense of demonstrating that there is no need for invoking other He II-ionizing sources beyond luminous quasars. The observed brightness of QSO 03020-0014 is sufficient for it to have created its surrounding He III zone

\footnotetext{
${ }^{3}$ Specifically, our statistical analysis of the STIS data reveals that the background signal on the STIS MAMA detectors displays a larger variance than can be accounted for by photon statistics alone. Consequently, we believe that the statistical error on the STIS null flux detection limit has been underestimated in previous work, and that the inferred He II optical depths in the troughs therefore must be considered to be strict lower limits.
}

"from scratch" in an IGM that had previously only been exposed to sources having a soft spectrum capable of ionizing H I and He $\mathrm{I}$ by $z \sim 6$, but which left the helium content locked in singly-ionized form.

This issue of the uncertain initial conditions aside, another central underlying assumption implicit in the Smette et al. (2002) model for the opacity gaps is that the intergalactic medium in the immediate vicinity of a luminous quasar - and the $\mathrm{He}$ II/He III ratio in particular - is at all times in photoionization equilibrium with the instantaneous radiation field. That this is not a very realistic assumption for the problem at hand can be seen by considering the time scales for the two processes involved in determining the He II/He III ionization balance; photoionization of He II and recombination of He III.

From Fig. 2 and the photometry described above, we estimate the observed continuum flux of QSO 03020-0014 at Ly $\alpha$ to be $F_{\lambda} \simeq 3 \times 10^{-17} \mathrm{erg} \mathrm{s}^{-1} \mathrm{~cm}^{-2} \AA^{-1}$. For an assumed extreme ultraviolet spectrum $F_{v} \propto v^{-\alpha}$ with $\alpha \simeq 1.8$ (Telfer et al. 2002), the corresponding rest-frame luminosity of QSO 03020-0014 at the He II-ionization edge is $L_{v \mathrm{He} \mathrm{II}} \simeq 3 \times 10^{29} \mathrm{erg} \mathrm{s}^{-1} \mathrm{~Hz}^{-1}$. With this large a flux, the mean time between photoionizations experienced by $\mathrm{He}_{\mathrm{II}}$ ions situated at a distance $D_{\perp} \simeq 3.2 \mathrm{Mpc}$ from QSO 03020-0014 is very short:

$t_{i}=\Gamma_{\mathrm{He} \mathrm{II}}^{-1} \simeq\left(\frac{L_{v \mathrm{He} \mathrm{II}} \sigma_{\mathrm{He} \mathrm{II}}}{4 \pi D_{\perp}^{2} h(\alpha+3)}\right)^{-1} \simeq 3 \times 10^{6} \mathrm{yr}$

where $\sigma_{\text {He II }}=1.6 \times 10^{-18} \mathrm{~cm}^{2}$ is the He II photoionization cross section at threshold. In comparison, the mean time for He III recombination is extremely long, and comparable to the age of the Universe at $z \simeq 3.05$ :

$t_{\mathrm{r}} \simeq\left(\alpha_{\mathrm{He} \mathrm{III}} \bar{n}_{\mathrm{e}}\right)^{-1} \simeq 2 \times 10^{9} \mathrm{yr}$

where $\alpha_{\mathrm{He} \mathrm{III}}=1.6 \times 10^{-12} \mathrm{~cm}^{3} \mathrm{~s}^{-1}$ is the He III recombination coefficient and $\bar{n}_{\mathrm{e}} \simeq 1 \times 10^{-5} \mathrm{~cm}^{-3}$ is the mean electron density in the ionized IGM. Since $t_{\mathrm{r}} \gg t_{i}$, and $t_{\mathrm{r}} \gtrsim t_{\mathrm{Q}}$, it follows that intergalactic $\mathrm{He}_{\mathrm{II}}$ (and $\mathrm{H}_{\mathrm{I}}$ and $\mathrm{He}_{\mathrm{I}}$ for that matter) when exposed to a burst of ionizing radiation from a luminous quasar such as QSO 03020-0014 is unlikely to reach conditions of ionization equilibrium with photoionization balancing recombination on the time scale of interest. The IGM at $z \sim 3$ is, of course, not of uniform density. However, the fact that the recombination time is even longer in the underdense regions that fill the bulk of the volume and dominate the He II opacity (Miralda-Escudé et al. 2000) only strengthens the argument against the assumption of photoionization equilibrium, except in the extreme high density regions. In this case, (re)ionization is effectively a one-way process, and the key parameter determining the extent of the ionized region surrounding the quasar is not the instantaneous rate of ionizing photons, but rather the total dose accumulated over time.

The fourth time scale of interest is therefore the time required for QSO 03020-0014 to emit one $E>54 \mathrm{eV}$ photon for every helium atom contained within a cone reaching out to distance $D_{\perp}$ :

$t_{\gamma} \simeq \frac{4 \pi}{3} D_{\perp}^{3} \frac{\bar{n}_{\mathrm{He}}}{\dot{N}_{\mathrm{He} \mathrm{II}}} \simeq 5 \times 10^{6} \mathrm{yr}$ 
where $\dot{N}_{\mathrm{He} \mathrm{II}} \simeq L_{v \mathrm{He} \text { II }} / h \alpha \simeq 2 \times 10^{55}$ photons s$^{-1}$ is the total output of $E>54 \mathrm{eV} \mathrm{He}$ II-ionizing photons and $\bar{n}_{\mathrm{He}} \simeq$ $8 \times 10^{-7} \mathrm{~cm}^{-3}$ is the average density of intergalactic helium atoms. Since $t_{\gamma}<D_{\perp} / c \simeq 1 \times 10^{7} \mathrm{yr}$, it follows that QSO 03020-0014 at its observed brightness at $z \simeq 3.05$ could comfortably have created a He III region reaching out to the sight line to Q0302-003 in the time required - even if the He II is not pre-ionized at $z \sim 3$.

Note that the above timing and photon budget arguments are equally applicable to hydrogen. In particular, since the ratio of $E>13.6 \mathrm{eV}$ photons to $E>54 \mathrm{eV}$ photons emitted by a quasar having an extreme ultraviolet spectral index $\alpha \simeq 1.8$ is comparable to the ratio of hydrogen to helium atoms, the burst of H I-ionizing radiation from QSO 03020-0014 should have no trouble accounting for the dearth of Lyman forest lines seen at the position of the He II opacity gaps - especially since hydrogen was almost certainly already re-ionized at $z \sim 3$.

In light of the above considerations, a more realistic simple picture of the interaction of QSO 03020-0014 with the surrounding IGM is that its intense burst of ionizing radiation gives rise to a He III region that expands outward for the duration of the quasar life at a velocity given by

$$
v=\frac{\mathrm{d} R}{\mathrm{~d} t} \simeq \frac{\dot{N}_{\mathrm{He} \text { II }}}{4 \pi R^{2} n_{\mathrm{He} \mathrm{II}}^{0}} \quad v \leq c
$$

where $n_{\mathrm{He} \text { II }}^{0}$ is the initial He II density encountered in the given direction at the distance $R$ from the quasar, and it is understood that $\mathrm{d} R / \mathrm{d} t$ is bounded by the speed of light (Cen \& Haiman 2000).

Since the intergalactic medium at $z \sim 3$ is not uniform, but displays density variations spanning more than three orders of magnitude on the spatial scales of interest, it is clear that the ionization front from QSO 03020-0014 in reality will propagate outward in a highly complicated and inhomogeneous manner that depends sensitively on the initial conditions. At the observed brightness of QSO 03020-0014, the ionizing radiation will propagate to distances $R \simeq D_{\perp}$ at the speed of light in directions containing only gas of less than average density $n_{\mathrm{He} \text { II }}^{0} \lesssim 0.7 \bar{n}_{\mathrm{He}}$. Locations shadowed by optically thick high density regions, on the other hand, will be exposed to a lower total ionizing dose since the ionization front will arrive later due to it needing to first eat its way through the denser gas according to (7). Miralda-Escudé et al. (2000) describe how, when viewed on larger scales, this process leads to a gradual reionization of the IGM where the underdense regions are reionized first and the overdense regions last.

\subsection{Geometry}

As discussed by Smette et al. (2002), there is no reason to expect QSO 03020-0014 to irradiate its surroundings in an isotropic manner. According to the unified model for quasars and AGNs, the central massive black hole is surrounded by a dense optically thick molecular torus which confines the escaping radiation to a $\phi \sim 90^{\circ}$ wide ionization cone on each side of the torus (Barthel 1989). Such beaming can naturally explain why the He II opacity gap appears offset from the redshift of QSO 03020-0014.

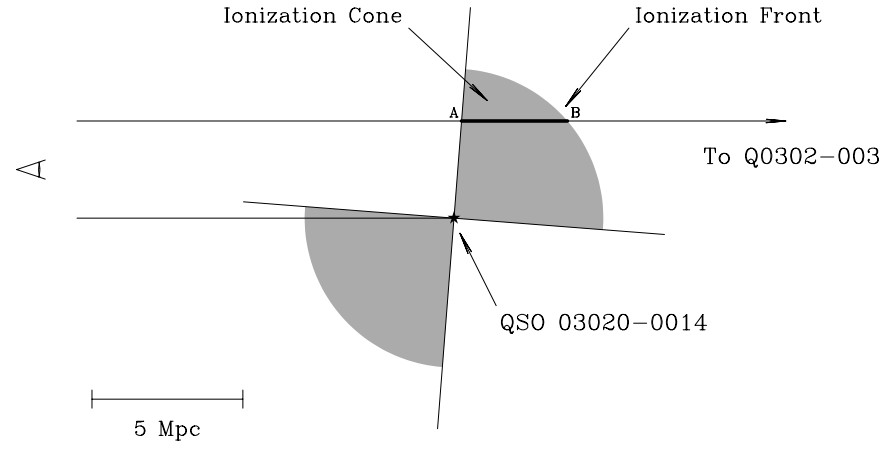

Fig. 4. Schematic of our proposed model for the geometry of the He III ionization region surrounding QSO 03020-0014 and its interception by the line of sight to Q0302-003. The extent of the He II opacity gap is indicated by the highlighted line. The scale refers to the epoch $z=3.05$.

From Fig. 3 we adopt $z_{\mathrm{A}} \simeq 3.051$ as the onset of the opacity gap and $z_{\mathrm{B}} \simeq 3.065$ as its apparent high redshift edge. For our adopted emission redshift of $z \simeq 3.050$ the He II gap sets in at a distance $D_{\mathrm{A}} \simeq 0.3 \mathrm{Mpc}$ behind the projected position of QSO 03020-0014 and stops at a distance $D_{\mathrm{B}} \simeq 3.8 \mathrm{Mpc}$ behind the quasar. This location of the opacity gap with respect to QSO 03020-0014 is drawn to scale in Fig. 4.

Also indicated in Fig. 4 is the orientation and extent of the radiation pattern from QSO 03020-0014 suggested by the above geometry. It is evident that the free parameters are tightly constrained. The orientation of the leftmost edge of the top ionization cone is set by the requirement that the sightline at $z \lesssim 3.051$ not be illuminated, while the radial extent of the He III zone is fixed by the requirement that the ionization front has not yet reached beyond the high redshift edge of the gap at $z \gtrsim 3.065$. Lastly, the requirement that we be able to see and recognize QSO 03020-0014 as a quasar through the opposing light cone constrains the opening angle to $\phi \gtrsim 85^{\circ}$ (assuming the top and bottom cones to be symmetrical). Although admittedly speculative, it is tempting to interpret the strong $z_{\mathrm{abs}} \approx z_{\mathrm{em}}$ absorption detected in our spectrum of QSO 03020-0014 as evidence that we are indeed viewing the quasar along a line of sight that grazes the inner edge of its ionization cone.

At the next level of detail, it is of interest to explore the approximate shape of the opacity gap anticipated in our proposed model. In particular, it is worth probing whether it might be possible to constrain the initial ionization state of the intergalactic He II from the observed profile shape, and thereby address the outstanding issue of the redshift at which helium reionization occurred.

It is illustrative to first consider the simplest case of an He III ionization front that propagates at the constant speed of light over the distances of interest. A key feature of the geometry shown in Fig. 4 is that different locations along the line of sight through the opacity gap are not exposed to the same dose of ionizing radiation at any given time. The ionization front first reached the line of sight to Q0302-003 at position $\mathrm{A}$ at the near side of the opacity gap at a time $t_{\mathrm{A}}=R_{\mathrm{A}} / c=\left(D_{\perp}^{2}+D_{\mathrm{A}}^{2}\right)^{\frac{1}{2}} / c \simeq$ $1.1 \times 10^{7} \mathrm{yr}$ after QSO 03020-0014 first turned on. As the ionization front propagated further outward, progressively more 
distant segments of the sight line become illuminated. The back end of the gap marks the progression of the ionization front at the time $t_{\mathrm{B}}=R_{\mathrm{B}} / c=\left(D_{\perp}^{2}+D_{\mathrm{B}}^{2}\right)^{\frac{1}{2}} / c \simeq 1.6 \times 10^{7} \mathrm{yr}$ after turn on. Since the nearer side of the gap is observed at the later epoch $\tilde{t}_{\mathrm{A}}=t_{\mathrm{B}}+\left(D_{\mathrm{B}}-D_{\mathrm{A}}\right) / c \simeq 2.8 \times 10^{7} \mathrm{yr}$, it follows that at the time of observation the He II gas at this position had been exposed to the ionizing flux from QSO 03020-0014 for the duration $\Delta t_{\mathrm{E}}^{\max } \simeq \tilde{t}_{\mathrm{A}}-t_{\mathrm{A}} \simeq 1.7 \times 10^{7} \mathrm{yr}$. This value of $\Delta t_{\mathrm{E}}^{\max }$ becomes larger if the expansion occurs at sub-luminal speed as per (7) since more time is required for the ionization front to propagate from $R_{\mathrm{A}}$ to $R_{\mathrm{B}}$. Clearly, the typical total exposure time decreases through the opacity gap from $\Delta t_{\mathrm{E}}=\Delta t_{\mathrm{E}}^{\max }$ at the near end at point $\mathrm{A}$ to $\Delta t_{\mathrm{E}}=0$ at the far end at point $\mathrm{B}$.

In our proposed geometry, the low redshift edge of the gap reflects the angular cut-off of the quasar radiation cone shaped by the central torus, which is unlikely to be perfectly sharp as assumed above, and could well vary in time. Taking such a gradual extinction of the ionizing flux into account will make the onset of the gap less sharp and shift its peak to higher redshift as observed. It is interesting to note that had the radiation from QSO 03020-0014 not been hindered in illuminating the front $z \lesssim 3.05$ portion of the line of sight as in our interpretation, but radiated the line of sight isotropically, the significant time delay effects would cause the opacity gap to extend considerably further in front of the quasar than behind it.

When recombination can be ignored, the residual density of He II ions following an exposure of duration $\Delta t_{\mathrm{E}}$ is simply

$n_{\mathrm{He} \mathrm{II}}\left(\Delta t_{\mathrm{E}}\right) \simeq n_{\mathrm{He} \mathrm{II}}^{0} \mathrm{e}^{-\Delta t_{\mathrm{E}} / \bar{t}_{i}}$

where $n_{\mathrm{He} \text { II }}^{0}$ is the initial He II density and the mean ionization time $\bar{t}_{i}$ is the reciprocal of the local ionization rate averaged over $\Delta t_{\mathrm{E}}$

$\bar{t}_{i} \simeq \bar{\Gamma}_{\mathrm{He} \text { II }}^{-1}=\left(\frac{1}{\Delta t_{\mathrm{E}}} \int_{\Delta t_{\mathrm{E}}} \Gamma_{\mathrm{He} \text { II }} \mathrm{d} t\right)^{-1}$.

Note that since to order of magnitude we have $\Delta t_{\mathrm{E}} \sim D_{\perp} / c \sim$ $10^{7} \mathrm{yr}$ and $\bar{t}_{i} \sim t_{i} \sim 10^{6} \mathrm{yr}$ from (4), we are clearly in a regime where the residual density of $\mathrm{He}$ II - and thereby the observable optical depth $\tau_{\text {He II }}-$ is extremely sensitive to even minute changes in the ratio $\Delta t_{\mathrm{E}} / \bar{t}_{i}$. Note also that the time average in (9) refers to the unknown photon output of QSO 03020-0014 during a time interval prior to our observing it at $z \simeq 3.05$.

With these caveats in mind, it is instructive to consider the simplest case of a constant quasar luminosity and an IGM of uniform density. In this model $\bar{t}_{i}$ at a given point on the line of sight is approximately given by (4) with $R$ substituted for $D_{\perp}$, and the observed optical depth corresponding to a given residual He II density is given by the familiar Gunn-Peterson expression

$\tau_{\mathrm{He} \text { II }}=\left(\frac{c}{H_{0}}\right) \frac{n_{\mathrm{He} \mathrm{II}}\left(\Delta t_{\mathrm{E}}\right) \sigma_{l}}{\left(\Omega_{0}(1+z)^{3}+\Lambda_{0}\right)^{\frac{1}{2}}}$

where $\sigma_{l}=\lambda_{l} \frac{\pi e^{2}}{m_{\mathrm{e}} c^{2}} f_{i j}=1.1 \times 10^{-18} \mathrm{~cm}^{2}$ is the integrated cross section of the He II $\lambda 304$ transition.

In Fig. 5 we plot the predicted gap profiles for three different combinations of $n_{\mathrm{He} \text { II }}^{0}$ and $R_{\mathrm{B}}$, all calculated assuming

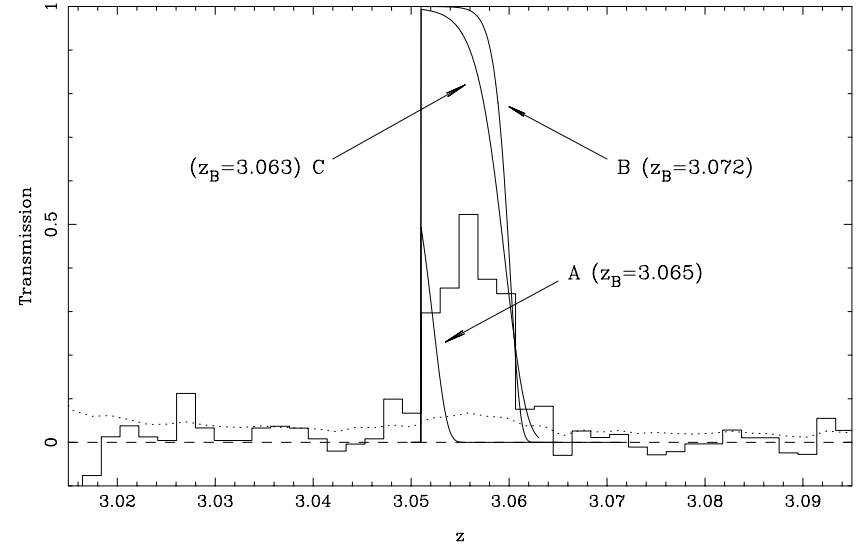

Fig. 5. Predicted He II absorption profiles in the case of a smooth IGM and a constant quasar output. The labels refer to the assumed value of $z_{\mathrm{B}}$ and the initial He II optical depth (see text).

QSO 03020-0014 to have radiated at its observed brightness at $z \simeq 3.05$ since turn on, and using (7) to calculate $R(t)$ and $\Delta t_{\mathrm{E}}$. Case $\mathrm{A}$ assumes the value $z_{\mathrm{B}} \simeq 3.065$ from above and $n_{\mathrm{He} \mathrm{II}}^{0}=\bar{n}_{\mathrm{He}} \simeq 8 \times 10^{-7} \mathrm{~cm}^{-3}$, corresponding to the fully delayed He II reionization scenario. It is seen that the resulting profile reproduces the $\sim 50 \%$ flux decrement of the gap, but not its width due to the rapid drop-off of the ionizing dose along the line of sight. However, since in this case the quasar has to create its He III zone in a medium having a huge initial Gunn-Peterson optical depth of $\tau_{\mathrm{He} \text { II }}^{0} \simeq 2.9 \times 10^{3}$, the difference between a gap having $\tau_{\mathrm{He} \text { II }} \simeq 1$ and a completely transparent $\left(\tau_{\mathrm{He} \mathrm{II}} \ll 1\right)$ opacity gap represents a minute fraction of the total He II ionized that can easily be attributed to residual shielded He $\mathrm{II}$ in the very densest regions. The observable $z_{\mathrm{B}}$ is also in practice not necessarily the redshift at which the ionization front reaches the most remote part of the line of sight, but rather the redshift at which the He II optical depth of the gap has diminished to a sufficiently small value for the attenuated quasar flux to rise above the detection limit. As shown by the second example B in Fig. 5, if one does not attempt to fit the finite depth of the gap, its width can be reproduced simply by increasing this parameter to $z_{\mathrm{B}} \simeq 3.072-$ i.e. by allowing QSO 03020-0014 to turn on earlier. Example A implicitly assumes the quasar to have turned on at $z \simeq 3.09$, and having shone for $t_{\mathrm{Q}} \simeq 4 \times 10^{7} \mathrm{yr}$ prior to our observing it at $z \simeq 3.05$. In case $\mathrm{B}$ the quasar instead turns on at $z \simeq 3.13$, which lengthens the life time to $t_{\mathrm{Q}} \simeq 6 \times 10^{7} \mathrm{yr}$ and increases the total emitted radiation dose correspondingly. In other words, even without evoking variations in the brightness of QSO 03020-0014, the total accumulated ionizing radiation dose can be matched to any assumed initial value of $n_{\mathrm{He} \text { II }}^{0}$ simply by adjusting the age of the quasar. This is illustrated in example $\mathrm{C}$, where $z_{\mathrm{B}} \simeq 3.063$ has been chosen to match the gap width in the other extreme "pre-ionized" case $n_{\mathrm{He} \text { II }}^{0} \simeq 1 \times 10^{-9} \mathrm{~cm}^{-3}$ corresponding to $\tau_{\mathrm{He} \mathrm{II}}^{0} \simeq 4.5$ as assumed by Smette et al. (2002).

Since profiles B and C for our purposes are indistinguishable, this suggests that more sophisticated modelling will also not be able to infer the value of $n_{\mathrm{He} \mathrm{II}}^{0}$ from the He II gap profile alone. It is interesting, however, to speculate whether it may be possible to distinguish between cases $\mathrm{B}$ and $\mathrm{C}$ by also 
considering the $\mathrm{H}$ I-ionizing radiation from QSO 03020-0014. Since the intergalactic hydrogen is pre-ionized at $z \sim 3$ in both cases, the additional $E>13.6 \mathrm{eV}$ flux from QSO 03020-0014 in the fully delayed He II reionization scenario is expected to reach the line of sight to Q0302-002 faster than the $E>54 \mathrm{eV}$ flux, in which case the "transverse" proximity effect on the $\mathrm{H}_{\mathrm{I}}$ Lyman forest would be expected to extend to higher redshift than the He II opacity gap. The Keck spectrum of Q0302-002 shown in Fig. 15 of Heap et al. (2000) does show several weak Ly $\alpha$ lines in the $z \simeq 3.06-3.07$ range, but there is, of course, no way of knowing whether these systems were high column density systems prior to being illuminated by QSO 03020-0014 just as there is no way of knowing when QSO 03020-0014 actually turned on and how bright it was prior to the epoch of observation.

In conclusion, the modelling above, while naive, does serve to demonstrate that the need to abandon the convenient assumption of photoionization equilibrium adds several additional unknowns to the problem of the He II gaps, making them exceedingly difficult to interpret in detail - beyond the simple photon budget and timing considerations of Eqs. (6) and (7).

\section{Summary and conclusions}

We have identified the adjacent quasar which is almost certainly responsible for creating the isolated low opacity gap reported by Heap et al (2000) at $z \simeq 3.056$ in the STIS He II absorption spectrum of Q0302-003. The object, QSO 03020-0014, lies at a distance $\theta=6.5\left(D_{\perp} \simeq 3.2 \mathrm{Mpc}\right)$ from Q0302-003, and was uncovered by means of a dedicated search of a $13^{\prime} \times 13^{\prime}$ field centered on Q0302-003 using FORS on the VLT in a filtered slitless spectroscopic mode. Our follow-up FORS spectroscopy reveals the redshift of QSO $03020-0014$ to be $z \simeq 3.050 \pm 0.003$, which places it close to the near edge of the Q0302-003 opacity gap in redshift.

We have presented a plausible model for the shape of the He III ionization zone surrounding QSO 03020-0014. That QSO 03020-0014 lies slightly in front of the opacity gap in redshift is interpreted to imply that the He II-ionizing flux from QSO 03020-0014 is confined to two opposing cones as suggested by unified models of AGNs and quasars. One cone is aimed at the line of sight to Q0302-003 and the object is orientated such that it can be seen and recognized as a quasar from Earth through its opposing light cone.

We have also pointed out that the customary assumption of photoionization equilibrium is very unlikely to be applicable in the rarefied IGM exposed to the intense and transient radiation field of a bright quasar such as QSO 03020-0014. In particular, since the He III recombination time is much longer than the quasar lifetime in all but the most overdense regions of the IGM, the primary factors determining the extent of the He III zone surrounding a quasar is the initial density of He II ions and the total integrated output of He II-ionizing photons emitted since the quasar first turned on. Both parameters are unknown but at its observed brightness of $V \simeq 20.5$ at $z \simeq 3.05$, QSO 03020-0014 could comfortably have ionized a region large enough to explain the He II opacity gap seen toward
Q0302-003 in $t_{Q} \simeq 5 \times 10^{7} \mathrm{yr}-$ even in the extreme delayed helium reionization case where the IGM was first reionized by soft spectrum sources at $z \sim 6$ leaving all the intergalactic helium locked up in the form of He II at $z \simeq 3$.

Our interpretation of the geometry and physics of the QSO 03020-0014 + Q0302-003 system may not bode too well for the prospect of identifying the neighboring quasars giving rise to the He II opacity gaps detected toward other lines of sight. Because of the long recombination time scale, some gaps may well be "fossil" He III regions for which the associated quasar has already turned off. Even if the quasar causing a gap is still active, we may still not detect and recognize it as such if the light is confined to an ionization cone orientated perpendicular to the line of sight. We are presently extending our search technique to the He II gaps seen toward HE 2347-4342 and PKS 1935-692, the outcome of which will reveal how unique QSO 03020-0014 really is.

Acknowledgements. The staff of the Paranal Observatory are thanked for their helpful and professional assistance in carrying out the VLT observations described in this paper.

\section{References}

Adelberger, K. L., Steidel, C. C., Shapely, A. E., \& Pettini, M. 2002, ApJ, in press [astro-ph/0210314]

Anderson, S. F., Hogan, C. J., Williams, B. F., \& Carswell, R. F. 1999, AJ, 117, 56

Barger, A. J., Cowie, L. L., Bautz, et al. 2001, AJ, 122, 2177

Barthel, P. D. 1989, ApJ, 336, 606

Becker, R. H., Fan, X., White, R. L., et al. 2001, AJ, 122, 2850

Böhnhardt, H. (ed.) 2000, FORS1+2 User Manual, VLT-MAN-ESO-13100-1543/2.2

Cen, R., \& Haiman, Z. 2000, ApJ, 542, L75

Davidsen, A. F., Kriss, G. A., \& Zheng, W. 1996, Nature, 380, 47

Djorgovski, S. G., Castro, S. M., Stern, D., \& Mahabal, A. A. 2001, ApJ, 560, L5

Gaskell, C. M. 1982, ApJ, 263, 79

Haiman, Z., \& Hui, L. 2001, ApJ, 547, 27

Hamuy, M., Suntzeff, N. B., Heathcote, S. R., et al. 1994, PASP, 106, 566

Heap, S. R., Williger, G. M., Smette, A., et al. 2000, ApJ, 534, 69

Hogan, C. J., Anderson, S. F., \& Rugers, M. H. 1997, AJ, 113, 1495

Jakobsen, P. 1998, A\&A, 331, 61

Jakobsen, P., Boksenberg, A., Deharveng, J. M., et al. 1994, Nature, 370, 35

Kriss, G. A., Shull, J. M., Oegerle, W., et al. 2001, Science, 293, 5532

Landolt, A. U. 1992, AJ, 104, 340

Martini, P., \& Weinberg, D. H. 2001, ApJ, 547, 12

McIntosh, D. H., Rix, H.-W., Rieke, M., \& Foltz, C. B. 1999, ApJ, 517, L73

Miralda-Escudé, J., Haehnelt, M., \& Rees, M. J. 2000, ApJ, 530, 1

Pei,Y. C. 1995, ApJ, 438, 623

Pen, U. 1999, ApJS, 120, 49

Pentericci, L., Fan, X., Rix, H. W., et al. 2002, ApJ, 123, 2151

Reimers, D., Köhler, S., Wisotzki, L., et al. 1997, A\&A, 327, 890

Seifert, W., Appenzeller, I., Fürtig, W., et al. 2000, Proc. SPIE, ed. M. Iye, \& A. F. Moorwood, 4008, 96

Smette, A., Heap, S. R., Williger, G. M., et al. 2002, ApJ, 654, 542

Telfer, R. C., Zheng, W., Kriss, G. A., \& Davidsen, A. F. 2002, ApJ, 565,773

Tytler, D., \& Fan, X. M. 1992, ApJS, 79, 1

Tytler, D., Fan, X. M., Burles, S., et al. 1995, in QSO Absorption Lines, ed. G. Meylan (Springer, Berlin), 289 